\title{
CROSSED PRODUCT ALGEBRAS ATTACHED TO WEIGHT ONE FORMS
}

\author{
Debargha Banerjee and Eknath Ghate
}

Abstract. We completely determine the Brauer class of the crossed product algebra attached to a non-dihedral cusp form of weight one.

\section{Introduction}

Let $f$ be a holomorphic cuspidal newform of weight at least one. In weights two and higher, there is a Grothendieck motive $M_{f}$ (abelian variety $A_{f}$ in weight two) attached to $f$. It is a fundamental fact, due to Momose and Ribet 8 ] in weight two and 1, [4 in higher weights, that the full algebra of endomorphisms of this motive has the structure of an explicit crossed product algebra $X$, when the form $f$ is of non-dihedral type. Moreover, much, but not all, is known about the Brauer class of this algebra [9], 6], 4].

In this paper, we completely determine the Brauer class of $X$ for non-dihedral cusp forms of weight one. The Brauer class in this case is closely related to the second Witt invariant of the trace form of a number field determined by the projective Artin representation associated to the form.

We show that, much as in higher weights, the Brauer class of $X$ is completely determined at a prime of good reduction by the parity of a certain slope, when this slope is finite (Theorem 22. An interpretation of this slope in terms of the adjoint representation allows us to compute it, showing that, in contrast to what happens in higher weights, the Brauer class is essentially unramified at all primes of good reduction (Theorem 4. Corollary 7). At primes of bad reduction we show that the Brauer class is determined purely by the nebentypus of the form (Theorem 5 . Corollary 7). Finally, as examples, we determine the Brauer class of $X$ for all non-dihedral weight one forms of prime level (Corollary 9).

\section{The crossed product algebra $X$}

The crossed product algebra $X$ we wish to study can be attached to non-dihedral forms in all weights. We recall the definition for forms of weight one.

Let $f=\sum a_{n} q^{n} \in S_{1}(N, \epsilon)$ be a normalized cuspidal newform of weight one, and nebentypus character $\epsilon$. Let $E=\mathbb{Q}\left(a_{n}\right)$ be the number field generated by the Fourier

Received by the editors October 28, 2009. Revision received November 25, 2010.

2000 Mathematics Subject Classification. Primary: 11F11, Secondary: 11F80, 11F30.

Key words and phrases. Modular forms, Brauer class. 
coefficients of $f$. Let $\rho_{f}$ be the finite image (Artin) Galois representation attached to $f$, as constructed in [2, Theorem 4.1], so

$$
\rho_{f}: G_{\mathbb{Q}} \rightarrow \mathrm{GL}_{2}(E)
$$

and $\operatorname{trace}\left(\rho_{f}\left(\operatorname{Frob}_{p}\right)\right)=a_{p}$ and $\operatorname{det}\left(\rho_{f}\left(\operatorname{Frob}_{p}\right)\right)=\epsilon(p)$, for all primes $p \nmid N$. That $\rho_{f}$ has a model over $E$ follows from the oddness of $\rho_{f}$ [2, p. 521, footnote]. We also note that the extension $E / \mathbb{Q}$ is abelian, since $E$ is generated by the traces of matrices of finite order. By a standard classification, the projective image of $\rho_{f}$ is either

- a dihedral group, or,

- $A_{4}, S_{4}$ or $A_{5}$.

In the last three cases we say $f$ is a non-dihedral form. More specifically, in these cases $f$ is said to be of tetrahedral, octahedral, or icosahedral type, respectively.

Let $f$ be of non-dihedral type. Let $\Gamma$ be the group of inner twists contained in $\operatorname{Aut}(E / \mathbb{Q})$, i.e.,

$$
\Gamma=\left\{\gamma \in \operatorname{Aut}(E) \mid f^{\gamma}=f \otimes \chi_{\gamma}, \text { for some } E^{*} \text {-valued Dirichlet character } \chi_{\gamma}\right\} .
$$

Define the $E$-valued 2-cocycle $c$ on $\Gamma$ by

$$
c(\gamma, \delta)=\frac{G\left(\chi_{\gamma}^{-1}\right) G\left(\chi_{\delta}^{-\gamma}\right)}{G\left(\chi_{\gamma \cdot \delta}^{-1}\right)} \in E^{*},
$$

for $\gamma, \delta \in \Gamma$. Let $X$ be the crossed product algebra associated to the cocycle $c(\gamma, \delta)$ defined by

$$
X=\bigoplus_{\gamma \in \Gamma} E \cdot x_{\gamma}
$$

where the $x_{\gamma}$ are formal symbols satisfying the relations

$$
\begin{aligned}
x_{\gamma} \cdot x_{\delta} & =c(\gamma, \delta) \cdot x_{\gamma \cdot \delta} \\
x_{\gamma} \cdot e & =\gamma(e) \cdot x_{\gamma},
\end{aligned}
$$

for $\gamma, \delta \in \Gamma, e \in E$. Then $X$ is a central simple algebra over $F$, the fixed field of $\Gamma$ in $E$. We note $F$ contains $a_{p}^{2} \epsilon^{-1}(p)$, for $p \nmid N$, and is the subfield of $E$ generated by these quantities.

\section{Brauer class of $X$}

In this section we study the Brauer class of $X$ as an element of the Brauer group of $F$.

3.1. Definition of $\alpha$. In weights bigger than one, a crucial role is played by a certain map $\alpha: G_{\mathbb{Q}} \rightarrow E^{*}$. We now define an analogue of this map in the weight one setting.

The map $\alpha$ in higher weights is defined directly on the geometric object $A_{f}$ or $M_{f}$, using the Skolem-Noether theorem [9, §2], 44, §4]. It seems difficult to extend this definition to weight one. However, a purely algebraic definition of $\alpha$ in higher weight was given by Papier (cf. [10, p. 192]), which extends nicely to the weight one setting, as follows.

For $\gamma \in \Gamma$, there is a unique Dirichlet character $\chi_{\gamma}$ such that $f^{\gamma}=f \otimes \chi_{\gamma}$, and hence

$$
\rho_{f^{\gamma}} \sim \rho_{f} \otimes \chi_{\gamma} .
$$


For $\gamma, \delta \in \Gamma$, the identity $\chi_{\gamma \delta}=\chi_{\gamma} \chi_{\delta}^{\gamma}$ shows that $\gamma \mapsto \chi_{\gamma}$ is a 1-cocycle. Specializing to $g \in G_{\mathbb{Q}}$, we see that $\gamma \mapsto \chi_{\gamma}(g)$ is a 1-cocycle as well. By Hilbert's theorem 90, $\mathrm{H}^{1}\left(\Gamma, E^{*}\right)$ is trivial, i.e., there is an element $\alpha(g) \in E^{*}$ such that

$$
\alpha(g)^{\gamma-1}=\chi_{\gamma}(g)
$$

for all $\gamma \in \Gamma$. Clearly, $\alpha(g)$ is completely determined up to multiplication by elements of $F^{*}$. Varying $g \in G_{\mathbb{Q}}$, we obtain a well defined map

$$
\tilde{\alpha}: G_{\mathbb{Q}} \rightarrow E^{*} / F^{*} .
$$

Since each $\chi_{\gamma}$ is a character, $\tilde{\alpha}$ is a homomorphism. We can now easily verify all the relevant properties of $\alpha$ which hold in higher weight ([7, Theorem 1.1] and [11, Theorem 5.5]), though the proofs are a bit different.

Lemma 1. The homomorphism $\tilde{\alpha}$ satisfies:

(1) $\tilde{\alpha}$ is unramified at all primes $p \nmid N$.

(2) For all $g \in G_{\mathbb{Q}}$, we have $\alpha^{2}(g) \equiv \epsilon(g) \bmod F^{*}$.

(3) If $a_{p} \neq 0$, then $\alpha\left(\right.$ Frob $\left._{p}\right) \equiv a_{p} \bmod F^{*}$.

Proof. (1) Since both $\rho_{f^{\gamma}}$ and $\rho_{f}$ are unramified at $p \nmid N$, we have $\chi_{\gamma}(g)=1$ for all $g \in I_{p}$, by display (2). The relation (3) shows that $\alpha(g) \in F^{*}$, for all $g \in I_{p}$, so $\tilde{\alpha}$ is unramified at $p \nmid N$.

(2) Taking determinants in display $\left[2\right.$, we have $\chi_{\gamma}^{2}=\epsilon^{\gamma-1}$. Again by equation (3) we have $\alpha^{2} \equiv \epsilon \bmod F^{*}$.

(3) If the trace of $\rho_{f}(g)$ for $g \in G_{\mathbb{Q}}$ is non-zero, then display (2) and equation (3) show that $\alpha(g) \equiv \operatorname{trace}\left(\rho_{f}(g)\right) \bmod F^{*}$. In particular, if $p \nmid N$ and $a_{p} \neq 0$, then $\alpha\left(\operatorname{Frob}_{p}\right) \equiv a_{p} \bmod F^{*}$.

3.2. The 2-cocycle undelying $X$. Let $G_{F}=\operatorname{Gal}(\overline{\mathbb{Q}} / F)$. By $[9$, Proposition 1 , p. 9 ], whose proof also holds in weight one, the class of $X$ in $\operatorname{Br}(F)=\mathrm{H}^{2}\left(G_{F}, \overline{\mathbb{Q}}^{*}\right)$ is given by the 2-cocycle

$$
(g, h) \mapsto \chi_{g}(h),
$$

for $g, h \in G_{F}$, where $\chi_{g}:=\chi_{\gamma}$, for $\gamma$ the image of $g$ in $\Gamma$. By (3), this 2-cocycle is the same as the 2-cocycle given by

$$
(g, h) \mapsto \frac{\alpha(h)^{g}}{\alpha(h)},
$$

which in turn differs from the 2-cocycle

$$
c(g, h)=\frac{\alpha(g) \alpha(h)}{\alpha(g h)}
$$

by a coboundary. Hence, the class of $X$ is given by the 2-cocycle $c(g, h)$ above.

Observe that the class of $c$ is independent of the lift $\alpha$ of $\tilde{\alpha}$. Suppose $\alpha^{\prime}$ is another lift of $\tilde{\alpha}$. Then $\alpha^{\prime}(g)=\alpha(g) \cdot f(g)$, for some map $f: G_{F} \rightarrow F^{*}$. Let us denote the 2-cocycle obtained from $\alpha^{\prime}$ by $c^{\prime}$. Then $c$ and $c^{\prime}$ differ by the map $(g, h) \mapsto \frac{f(g) f(h)}{f(g h)}$, which is clearly a 2-coboundary. 
We also note that the class of $c$ (hence $X$ ) is 2-torsion in the Brauer group of $F$. This follows immediately from part (2) of Lemma 1, noting that $c^{2}(g, h)=d(g) d(h) / d(g h)$ is a 2-coboundary, since $d(g):=\alpha^{2}(g) / \epsilon(g) \in F^{*}$.

3.3. Local Brauer class. To study the Brauer class of $X$, it suffices, as usual, to specify the Brauer class of $X_{v}:=X \otimes_{F} F_{v}$ in $\operatorname{Br}\left(F_{v}\right)$, for each place $v$ of $F$, where $F_{v}$ is the completion of $F$ at $v$. The case of infinite $v$ will be treated shortly. It is well-known that if $v$ is finite, then

$$
\operatorname{inv}_{v}: \operatorname{Br}\left(F_{v}\right) \simeq \mathbb{Q} / \mathbb{Z}
$$

via the invariant map $\operatorname{inv}_{v}$ at $v$.

To aid in our computation of $\operatorname{inv}_{v}\left(X_{v}\right)$, for $v$ finite, it is useful to recall the explicit definition of the invariant map (cf. [13]), which we do now. Let $I_{v}$ be the inertia subgroup of $G_{F}$ at the prime $v$. Let $G_{v}^{\mathrm{un}}=\operatorname{Gal}\left(F_{v}^{\mathrm{nr}} / F_{v}\right)$ be the Galois group of $F_{v}^{\mathrm{nr}}$, the maximal unramified extension of $F_{v}$, over $F_{v}$. The inflation map

$$
\operatorname{Inf}: \mathrm{H}^{2}\left(G_{v}^{\mathrm{un}},\left(F_{v}^{\mathrm{nr}}\right)^{*}\right) \rightarrow \operatorname{Br}\left(F_{v}\right)
$$

is well-known to be an isomorphism. Now, the surjective valuation $v: F^{*} \rightarrow \mathbb{Z}$ can be extended uniquely to $\left(F_{v}^{\mathrm{nr}}\right)^{*}$ which we continue to call $v$. This gives rise to a map

$$
v: \mathrm{H}^{2}\left(G_{v}^{\mathrm{un}},\left(F_{v}^{\mathrm{nr}}\right)^{*}\right) \rightarrow \mathrm{H}^{2}\left(G_{v}^{\mathrm{un}}, \mathbb{Z}\right)
$$

which we again denote by $v$. Also, the short exact sequence of abelian groups $0 \rightarrow \mathbb{Z} \rightarrow \mathbb{Q} \rightarrow \mathbb{Q} / \mathbb{Z} \rightarrow 0$ gives rise to a long exact sequence of cohomology groups, with boundary map

$$
\delta: \mathrm{H}^{1}\left(G_{v}^{\text {un }}, \mathbb{Q} / \mathbb{Z}\right) \rightarrow \mathrm{H}^{2}\left(G_{v}^{\text {un }}, \mathbb{Z}\right),
$$

which is an isomorphism, since $\mathrm{H}^{i}\left(G_{v}^{\mathrm{un}}, \mathbb{Q}\right)=0$, for $i=1,2$. We recall the definition of $\delta$. If $\chi: G_{v}^{\text {un }} \rightarrow \mathbb{Q} / \mathbb{Z}$ is a homomorphism, and $\tilde{\chi}$ is a lift of $\chi$ to $\mathbb{Q}$, then $\delta(\chi)$ is the $\mathbb{Z}$-valued 2-cocycle on $G_{v}^{\text {un }}$ given by

$$
(g, h) \mapsto \frac{\tilde{\chi}(g) \tilde{\chi}(h)}{\tilde{\chi}(g h)} .
$$

Finally, there is a map, say Ev (for evaluation)

$$
\mathrm{Ev}: \mathrm{H}^{1}\left(G_{v}^{\mathrm{un}}, \mathbb{Q} / \mathbb{Z}\right) \rightarrow \mathbb{Q} / \mathbb{Z}
$$

obtained by evaluating a homomorphism at the Frobenius at $v$. Then, by definition, the invariant map at $v$ is given by

$$
\operatorname{inv}_{v}=\operatorname{Ev} \circ \delta^{-1} \circ v \cdot \operatorname{Inf}^{-1}: \operatorname{Br}\left(F_{v}\right) \rightarrow \mathbb{Q} / \mathbb{Z} .
$$

Since the class of $X$ is 2-torsion in $\operatorname{Br}(F)$, the class of $X_{v}$ is also 2-torsion in $\operatorname{Br}\left(F_{v}\right)$, so its image under $\operatorname{inv}_{v}$ will be in $\frac{1}{2} \mathbb{Z} / \mathbb{Z}$. Identifying this group with $\mathbb{Z} / 2$, we see that $X_{v}$ is a matrix algebra over $F_{v}$ if $\operatorname{inv}_{v}\left(X_{v}\right)=0 \bmod 2$, and is a matrix algebra over a quaternion division algebra over $F_{v}$ if $\operatorname{inv}_{v}\left(X_{v}\right)=1 \bmod 2$. 
3.4. Good primes. We can now calculate the local Brauer class $X_{v}$ at a prime $v \mid p$ with $p \nmid N$ (good prime), using the explicit definition of the invariant map just given. For each such $v$, define

$$
m_{v}:=\left[F_{v}: \mathbb{Q}_{p}\right] \cdot v\left(a_{p}^{2} \epsilon^{-1}(p)\right) \in \mathbb{Z} \cup\{\infty\}
$$

where $v$ is now normalized so that $v(p)=1$.

The following theorem relating the ramification of $X_{v}$ to the parity of $m_{v}$, when it is finite, is the weight one analogue of [4, Theorem 2.2] and [1, Theorem 1.0.4]. Although we will shortly show that in weight one, $m_{v}=0$ almost always, when it is finite, we still spend some time on the proof of the theorem here, since it is simple, works in all weights, and includes the case $p=2$.

Theorem 2. Let $p \nmid N$ be a prime with $a_{p} \neq 0$, and let $v$ be a place of $F$ lying over $p$. Then $\operatorname{inv}_{v}\left(X_{v}\right)=m_{v}$ mod 2. Thus $X_{v}$ is a matrix algebra over $F_{v}$ if and only if the normalized slope $\left[F_{v}: \mathbb{Q}_{p}\right] \cdot v\left(a_{p}^{2} \epsilon^{-1}(p)\right)$ is even.

Proof. $X_{v}$ corresponds to the restriction of the 2-cocycle $c$ in 4 to $G_{v}:=\operatorname{Gal}\left(\bar{F}_{v} / F_{v}\right)$. We compute $\operatorname{inv}_{v}(c)=\left(\operatorname{Ev} \circ \delta^{-1} \circ v \circ \operatorname{Inf}^{-1}\right)(c)$ step by step.

Since inflation is an isomorphism there exists a 2-cocycle $\tilde{c}$ on $G_{v} / I_{v}$ such that

$$
\tilde{c}\left(g I_{v}, h I_{v}\right)=c(g, h)
$$

up to a 2-coboundary. In fact, if we take the above equality to be the definition of $\tilde{c}$, then it is easy to check that $\tilde{c}$ is a 2-cocycle on $G_{v} / I_{v}$, since $c$ is a 2-cocycle, and that it is well defined. Indeed, since $\tilde{\alpha}$ is unramified at $p$ (cf. part (1) of Lemma 1), we have $\tilde{\alpha}(g i)=\tilde{\alpha}(g) \tilde{\alpha}(i)=\tilde{\alpha}(g)$, for $g \in G_{v}$ and $i \in I_{v}$. Since we may replace $\alpha$ by any lift of $\tilde{\alpha}$, we may assume $\alpha(g i)=\alpha(g)$, for $g \in G_{v}$ and $i \in I_{v}$. Then, for $g, h \in G_{v}$ and $i, j \in I_{v}$, the quantities $c(g, h)$ and $c(g i, h j)$ coincide, and the cocycle $\tilde{c}\left(g I_{v}, h I_{v}\right)$ is independent of the choice of the representatives. Clearly the image of this particular $\tilde{c}$ under the inflation map is $c$. Now, by definition, $v(\tilde{c})$ is the 2 -cocycle induced by

$$
(g, h) \mapsto v\left(\frac{\alpha(g) \alpha(h)}{\alpha(g h)}\right)
$$

for $g, h \in G_{F}$. Recall $d(g)=\frac{\alpha^{2}(g)}{\epsilon(g)}$, for $g \in G_{F}$. Note $d(g) \in F^{*}$ by part (2) of Lemma 1. Then the 2-cocycle above is the same as the 2-cocycle induced by

$$
(g, h) \mapsto \frac{1}{2} \cdot v\left(\frac{d(g) d(h)}{d(g h)}\right) .
$$

Consider now the map $\chi: \operatorname{Gal}\left(F_{v}^{\mathrm{nr}} / F_{v}\right) \rightarrow \mathbb{Q} / \mathbb{Z}$ defined by

$$
\chi(g)=\frac{1}{2} \cdot v(d(g)) \bmod \mathbb{Z} .
$$

Under the map $\delta$ the 1-cocycle $\chi$ maps to the 2-cocycle above, so $\left(\delta^{-1} \circ v \circ \operatorname{Inf}^{-1}\right)(c)$ is just $\chi$. Hence

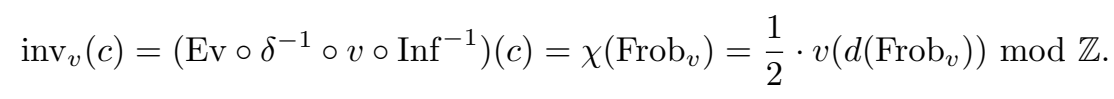


Let $e_{v}$ and $f_{v}$ be the ramification index and residue degree of $F_{v} / \mathbb{Q}_{p}$. Now Frob ${ }_{v}$ is the $f_{v}$-th power of $\operatorname{Frob}_{p}$, so we have

$$
\operatorname{inv}_{v}(c)=\frac{1}{2} \cdot f_{v} \cdot v\left(d\left(\operatorname{Frob}_{p}\right)\right) \bmod \mathbb{Z}
$$

And, by part (3) of Lemma 1, we have $\alpha\left(\operatorname{Frob}_{\mathrm{p}}\right) \equiv a_{p} \bmod F^{*}$, so that $d\left(\operatorname{Frob}_{p}\right)=$ $a_{p}^{2} / \epsilon(p) \bmod \left(F^{*}\right)^{2}$. It follows that

$$
\operatorname{inv}_{v}(c)=\frac{1}{2} \cdot f_{v} \cdot v\left(a_{p}^{2} / \epsilon(p)\right) \bmod \mathbb{Z} .
$$

Finally the valuation considered in the statement of the theorem is normalized so that $v(p)=1$, and differs from the valuation used in the proof by a factor of $e_{v}$. Noting $e_{v} f_{v}=\left[F_{v}: \mathbb{Q}_{p}\right]$, we obtain the theorem.

3.5. Adjoint representation. In this section we will compute the quantity $m_{v}$ appearing in Theorem 2 using the adjoint representation. This will allow us to essentially completely determine the Brauer class of the algebra $X$ at the good places $v \mid p$ with $a_{p} \neq 0$. The remaining good places $v$ for which $a_{p}$ vanishes are treated in the next section.

Recall that the adjoint representation

$$
\operatorname{Ad}\left(\rho_{f}\right): G_{\mathbb{Q}} \rightarrow \mathrm{GL}_{4}(E)
$$

attached to the representation $\rho_{f}: G_{\mathbb{Q}} \rightarrow \mathrm{GL}_{2}(E)$ is the 4-dimensional representation defined by the adjoint action:

$$
\operatorname{Ad}\left(\rho_{f}\right)(g)(X)=\rho_{f}(g) X \rho_{f}(g)^{-1},
$$

for $X \in \mathrm{M}_{2 \times 2}(E)$. It is important for us because of the following elementary but useful fact.

Lemma 3. Say $p \nmid N$. Then

$$
\operatorname{trace}\left(\operatorname{Ad}\left(\rho_{f}\right)\left(\operatorname{Frob}_{p}\right)\right)=\frac{a_{p}^{2}}{\epsilon(p)}
$$

Proof. If

$$
\rho_{f}\left(\operatorname{Frob}_{p}\right) \sim\left(\begin{array}{cc}
\alpha_{p} & 0 \\
0 & \beta_{p}
\end{array}\right),
$$

then a small computation shows that

$$
\operatorname{Ad}\left(\rho_{f}\right)\left(\operatorname{Frob}_{p}\right) \sim\left(\begin{array}{cccc}
1 & 0 & 0 & 0 \\
0 & \frac{\alpha_{p}}{\beta_{p}} & 0 & 0 \\
0 & 0 & 1 & 0 \\
0 & 0 & 0 & \frac{\beta_{p}}{\alpha_{p}}
\end{array}\right)
$$

So

$$
\operatorname{trace}\left(\operatorname{Ad}\left(\rho_{f}\right)\left(\operatorname{Frob}_{p}\right)\right)=2+\frac{\alpha_{p}}{\beta_{p}}+\frac{\beta_{p}}{\alpha_{p}}=\frac{\left(\alpha_{p}+\beta_{p}\right)^{2}}{\alpha_{p} \beta_{p}}=\frac{a_{p}^{2}}{\epsilon(p)}
$$


Theorem 4 (Good primes of finite slope). Let $f$ be a non-dihedral cuspidal newform in $S_{1}(N, \epsilon)$. Then

$X$ is unramified at $v$ for all good primes $v \mid p$ with $p \nmid N$ and $a_{p} \neq 0$, except possibly in the case when $p=2$ and the projective image of $\rho_{f}$ is $S_{4}$. Moreover, if the projective image of $\rho_{f}$ is

(1) $A_{4}$, then $F=\mathbb{Q}$,

(2) $S_{4}$, then $F=\mathbb{Q}$,

(3) $A_{5}$, then $F=\mathbb{Q}(\sqrt{5})$.

Proof. We have $\operatorname{Ad}\left(\rho_{f}\right)=\operatorname{Ad}^{0}\left(\rho_{f}\right) \oplus 1$, where $\operatorname{Ad}^{0}\left(\rho_{f}\right)$ is the irreducible 3-dimensional representation, afforded by the trace zero matrices.

Say the projective image of $\rho_{f}$ is $A_{4}$. From the character table of $A_{4}$, we see that $A_{4}$ has one 3-dimensional irreducible representation $V$, and three 1-dimensional representations (cf. [3, p. 20]). Thus

$$
\operatorname{Ad}\left(\rho_{f}\right)=V \oplus U
$$

where $U$ is the trivial representation. Computing the character of $V \oplus U$ on the four conjugacy classes of $A_{4}$, and using Lemma 3 above, we obtain

$$
\frac{a_{p}^{2}}{\epsilon(p)}=4,1,1 \text { or } 0 \text {. }
$$

Thus $F=\mathbb{Q}$ and by Theorem $2, X$ is unramified at all primes $v=p$, with $a_{p} \neq 0$ (note the 2 -adic valuation of 4 is even!).

Suppose now that the projective image of $\rho_{f}$ is $S_{4}$. From the character table of $S_{4}$ (cf. [3, p. 19]), we see that $S_{4}$ has two 1-dimensional representations, $U$ (trivial) and $U^{\prime}$ (sign), one 2-dimensional irreducible representation, and two 3-dimensional irreducible representations $V$ and $V \otimes U^{\prime}$. A small check shows that $\operatorname{Ad}^{0}\left(\rho_{f}\right)=V \otimes U^{\prime}$ (and not $V$ ), so that $\operatorname{Ad}\left(\rho_{f}\right)=\left(V \otimes U^{\prime}\right) \oplus U$. Computing traces on the 5 conjugacy classes as above, we get this time that

$$
\frac{a_{p}^{2}}{\epsilon(p)}=4,0,1,2 \text { or } 0
$$

So again, $F=\mathbb{Q}$, and if $v=p$ is a good odd prime with $a_{p} \neq 0$, then by Theorem 2 . $X$ is unramified at $v$. Note that since the 2-adic valuation of 2 is odd, $X$ may be ramified at the prime 2 (we shall later give examples where in fact $X_{2}$ is ramified).

Finally, let the projective image of $\rho_{f}$ be $A_{5}$. This time, there are two irreducible 3-dimensional representations $Y$ and $Z$, and so $\operatorname{Ad}\left(\rho_{f}\right)=Y \oplus U$ or $Z \oplus U$, where $U$ is the trivial representation. Again, computing traces on the 5 conjugacy classes using the character table of $A_{5}$ (cf. [3, p. 29]) we get

$$
\frac{a_{p}^{2}}{\epsilon(p)}=4,1,0, \frac{3 \pm \sqrt{5}}{2} \text { or } \frac{3 \mp \sqrt{5}}{2} \text {. }
$$

Since $F=\mathbb{Q}\left(\frac{a_{p}^{2}}{\epsilon(p)}\right)$, we conclude that $F=\mathbb{Q}(\sqrt{5})$. Now $\operatorname{Norm}_{\mathbb{Q}(\sqrt{5}) / \mathbb{Q}}\left(\frac{3 \pm \sqrt{5}}{2}\right)=1$, so that $\frac{3 \pm \sqrt{5}}{2}$ are units in $F$, and $v\left(\frac{3 \pm \sqrt{5}}{2}\right)=0$ for all primes $v$ of $F$. Again, by Theorem 2 , we conclude that $X_{v}$ is unramified for all good places $v$ of $F$ lying above $p$ with $a_{p} \neq 0$. 
3.6. Symbols. In this section we give formulas for the Brauer class of $X$ in terms of symbols, which will help us determine the Brauer class completely.

Let $\tilde{\rho}_{f}$ be the projectivization of $\rho_{f}$. Let $D_{K}$ be the discriminant of the unique quadratic extension $K / \mathbb{Q}$ in the $S_{4}$-number field cut out by $\tilde{\rho}_{f}$, when $\tilde{\rho}_{f}\left(G_{\mathbb{Q}}\right)=S_{4}$. Define the 2 -cocycle $c_{\epsilon}$ on $G_{\mathbb{Q}}$ by

$$
c_{\epsilon}(g, h)=\frac{\sqrt{\epsilon(g)} \sqrt{\epsilon(h)}}{\sqrt{\epsilon(g h)}},
$$

for $g, h \in G_{\mathbb{Q}}$. Then $\left[c_{\epsilon}\right]$ is 2-torsion in $\operatorname{Br}(\mathbb{Q})$. Let $\left[c_{\epsilon}\right]$ and $[X]$ also denote the images in $\operatorname{Br}(F)$ under the restriction map $\mathrm{H}^{2}\left(G_{\mathbb{Q}}, \bar{F}^{*}\right) \rightarrow \mathrm{H}^{2}\left(G_{F}, \bar{F}^{*}\right)$.

Theorem 5. The class of $X$ in $\operatorname{Br}(F)$ is as follows. If the image of $\tilde{\rho}_{f}$ is

(1) $A_{4}$, then $[X]=\left[c_{\epsilon}\right]$,

(2) $S_{4}$, then $[X]=\left[c_{\epsilon}\right] \cdot\left(2, D_{K}\right)$, where $(a, b)$ is the symbol for $F=\mathbb{Q}$,

(3) $A_{5}$, then $[X]=\left[c_{\epsilon}\right]$.

Proof. We use a general formula from [6] which works for weight one forms as well:

$$
[X]=\left[c_{\epsilon}\right] \cdot\left[c_{d}\right],
$$

where $\left[c_{\epsilon}\right] \in \operatorname{Br}(F)$ is as above, and $\left[c_{d}\right] \in \operatorname{Br}(F)$ is the product of the symbols

$$
\left[c_{d}\right]=\left(t_{1}, d_{1}\right) \cdots\left(t_{n}, d_{n}\right),
$$

where $t_{i}$ and $d_{i}$ are determined as follows. Note that $d=\alpha^{2} / \epsilon$ induces a continuous map

$$
d: G_{\mathbb{Q}} \rightarrow F^{*} / F^{* 2},
$$

where $F^{*} / F^{* 2}$ has the discrete topology. Thus $G_{\mathbb{Q}} / \operatorname{ker}(d) \simeq \operatorname{Gal}(K / \mathbb{Q}) \simeq(\mathbb{Z} / 2)^{m}$, for some elementary 2-extension $K$ of $\mathbb{Q}$. Now, for each $i$ between 1 and $m$, let $\sigma_{i} \in \operatorname{Gal}(K / \mathbb{Q})$ denote the element corresponding to $(0,0 . ., 1, . ., 0) \in(\mathbb{Z} / 2)^{m}$, with a 1 in the $i$-th spot. Then $t_{j} \in \mathbb{Q}^{*}$ is defined by $\sigma_{i}\left(\sqrt{t_{j}}\right)=(-1)^{\delta_{i j}} \sqrt{t_{j}}$. We lift each $\sigma_{i}$ to an element in $G_{\mathbb{Q}}$ which we continue to call $\sigma_{i}$, and set $d_{i}:=d\left(\sigma_{i}\right) \in F^{*} / F^{* 2}$.

We now claim that $\operatorname{ker}\left(\tilde{\rho}_{f}\right) \subset \operatorname{ker} d$. This is immediate, since if $\tilde{\rho}_{f}(g)=1$, for some $g \in G_{\mathbb{Q}}$, then $\rho_{f}(g)$ is a scalar, so $\operatorname{trace}\left(\rho_{f}(g)\right)^{2} / \operatorname{det}\left(\rho_{f}(g)\right)=4$. Since the trace is non-zero, this last expression is also equal to $\alpha^{2}(g) / \epsilon(g)$ up to an element of $F^{* 2}$, and hence $d(g)=1 \bmod F^{* 2}$, proving the claim. Thus, there is a surjection

$$
G_{\mathbb{Q}} / \operatorname{ker}\left(\tilde{\rho}_{f}\right) \rightarrow G_{\mathbb{Q}} / \operatorname{ker}(d) .
$$

The group on the left is $A_{4}, S_{4}$ or $A_{5}$, and the group on the right is an elementary 2-group. We conclude that in the $A_{4}$ and $A_{5}$ cases, the 2-group is trivial, and hence $d$ is trivial. This finishes the proof in these cases. In the $S_{4}$ case, the group on the right must be $\mathbb{Z} / 2$, or trivial. By our earlier computation of the character of the adjoint representation and the Chebotarev density theorem, there is a prime $p \nmid N$ such that $a_{p} / \sqrt{\epsilon(p)}= \pm \sqrt{2}$. By part (3) of Lemma 1, $d\left(\operatorname{Frob}_{p}\right)=2 \bmod F^{*}$ is non-trivial for such a prime $p$. Hence, the group on the right is $\mathbb{Z} / 2$. The resulting quadratic field cut out by $d$ must be $K$. Thus $m=1, t_{1}=D_{K}$, and $d_{1}=2$ up to a square in $F^{*}$. This shows that the class of $c_{d}$ is given by the symbol $\left(2, D_{K}\right)$, completing the proof in the $S_{4}$ case as well. 
3.7. Relation with Witt invariant. The Brauer class of the algebra $X$ is closely related to the 2 nd Witt invariant $w_{2}\left(Q_{K_{1}}\right) \in \operatorname{Br}(\mathbb{Q})$ of the quadratic form $Q_{K_{1}}(x)=$ $\operatorname{Tr}_{K_{1} / \mathbb{Q}}\left(x^{2}\right)$, studied in [14] and [5]. In the $A_{5}$ case, we let $w_{2}\left(Q_{K_{1}}\right)$ also denote the image of $w_{2}\left(Q_{K_{1}}\right)$ in $\operatorname{Br}(F)$ under the restriction map: $\mathrm{H}^{2}\left(G_{\mathbb{Q}}, \bar{F}^{*}\right) \rightarrow \mathrm{H}^{2}\left(G_{F}, \bar{F}^{*}\right)$. Here $K_{1}$ is a number field of degree $4\left(A_{4}\right.$ and $A_{5}$ cases $)$ or $6\left(A_{5}\right.$ case) with Galois closure equal to the fixed field of the kernel of the projective representation $\tilde{\rho}_{f}$ (and is determined up to isomorphism by this property).

Proposition 6. The Brauer class of $X$ is related to the image in $\operatorname{Br}(F)$ of the Witt invariant $w_{2}\left(Q_{K_{1}}\right)$ as follows. If the image of $\tilde{\rho}_{f}$ is

(1) $A_{4}$, then $[X]=w_{2}\left(Q_{K_{1}}\right)$,

(2) $S_{4}$, then $[X]=w_{2}\left(Q_{K_{1}}\right) \cdot\left(-1, D_{K}\right)$, where $(a, b)$ is the symbol for $F=\mathbb{Q}$,

(3) $A_{5}$, then $[X]=w_{2}\left(Q_{K_{1}}\right)$.

Proof. We use notation from [5. By Tate's theorem on p. 560 and the remark on p. 561 of loc. cit, we have $\rho^{*} \beta_{-}=\rho^{*} c_{2}=\epsilon^{*} c_{1}$ which is our $\left[c_{\epsilon}\right]$. The result now follows from [5. Theorem 3.6] and Theorem 5 above, noting that in the octahedral case, the discriminant of the quartic field $K_{1}$ differs from that of the quadratic field $K$ by a square.

3.8. Brauer class. We now state the following strengthening of Theorem 4, which includes the prime of good reduction for which $a_{p}$ vanishes, and gives complete information at the remaining primes as well.

Corollary 7 (Brauer class). $X$ is unramified at all primes of good reduction, except possibly in the $S_{4}$ case when $p=2$. At primes of bad reduction, the ramification of $X$ is controlled by the parity of the local factors of $\epsilon$. Finally, $X$ is ramified at all the infinite places.

Proof. It is known that $\left[c_{\epsilon}\right]$ is ramified at the infinite places since $\epsilon$ is odd, and at finite places $v \mid p$ of $F$, we have $\left[c_{\epsilon}\right]_{v}$ is trivial in $\operatorname{Br}\left(F_{v}\right)$ if and only if $\epsilon_{v}(-1)=1$ [ 6 . Moreover, in the $S_{4}$ case, $K$ is also easily checked to be the quadratic field cut out by the homomorphism $\epsilon: S_{4} \rightarrow \mathbb{Q}(\epsilon)^{*} / \mathbb{Q}(\epsilon)^{* 2}$ induced by the determinant of $\rho_{f}$, and $p \mid D_{K}$ implies $p \mid N$. The result now follows from Theorem 5 .

3.9. Prime level. As an example we completely determine the Brauer class of $X$ for forms of prime level. We have the following result [12, Theorem 7], which shows that only the octahedral and icosahedral cases occur in prime level.

Theorem 8. Say $f \in S_{1}(p, \epsilon)$ is a non-dihedral cuspidal newform of prime level $p$. Then

(a) $p$ is odd and $p \not \equiv 1 \bmod 8$

(b) If $p \equiv 5 \bmod 8$, then $\epsilon$ is of order 4 and the projective image of $\rho_{f}$ is $S_{4}$

(c) If $p \equiv 3 \bmod 4$, then $\epsilon$ is of order 2 and the projective image of $\rho_{f}$ is $S_{4}$ or $A_{5}$.

Following [12, we break case (c) into two cases (c1) and (c2), depending on whether the projective image of $\rho_{f}$ is $S_{4}$ or $A_{5}$. Theorem 5 gives: 
Corollary 9. For a weight one form of prime level $p$ as above, the Brauer class of $X$ in $\operatorname{Br}(F)$ is:

(b) $[X]=(-1,-2)$

(c1) $[X]=(-2,-p)$

(c2) $[X]=(-1,-p)$,

where $(a, b)$ is the symbol for $F$.

In particular, there are octahedral forms of odd level for which $X$ is ramified at the good prime 2 .

\section{Acknowledgements}

The first author would like to thank the second author for constant encouragement during the course of his Ph. D. at TIFR, and Prof. Jordi Quer for several valuable suggestions. The first author was partially supported by ARC grant DP0773301, and the second author by CEFIPRA-IFCPAR project 3701-2.

\section{References}

[1] A. F. Brown and E. P. Ghate, Endomorphism algebras of motives attached to elliptic modular forms, Ann. Inst. Fourier (Grenoble) 53 (2003), no. 6, 1615-1676.

[2] P. Deligne and J.-P. Serre, Formes modulaires de poids 1, Ann. Sci. École Norm. Sup. (4) 7 (1974) 507-530 (1975).

[3] W. Fulton and J. Harris, Representation theory, Vol. 129 of Graduate Texts in Mathematics, Springer-Verlag, New York (1991).

[4] E. Ghate, E. González-Jiménez, and J. Quer, On the Brauer class of modular endomorphism algebras, Int. Math. Res. Not. (2005), no. 12, 701-723.

[5] J. Quer, Liftings of projective 2-dimensional Galois representations and embedding problems, J. Algebra 171 (1995), no. 2, 541-566.

[6] - La classe de Brauer de l'algèbre d'endomorphismes d'une variété abélienne modulaire, C. R. Acad. Sci. Paris Sér. I Math. 327 (1998), no. 3, 227-230.

[7] K. A. Ribet, Endomorphisms of semi-stable abelian varieties over number fields, Ann. Math. (2) 101 (1975) 555-562.

[8] - Twists of modular forms and endomorphisms of abelian varieties, Math. Ann. 253 (1980), no. 1, 43-62.

[9] - Endomorphism algebras of abelian varieties attached to newforms of weight 2, in Seminar on Number Theory, Paris 1979-80, Vol. 12 of Progr. Math., 263-276, Birkhäuser Boston, Mass. (1981).

[10] - On l-adic representations attached to modular forms. II, Glasgow Math. J. 27 (1985) 185-194.

[11] - Abelian varieties over $\mathbf{Q}$ and modular forms, in Modular curves and abelian varieties, Vol. 224 of Progr. Math., 241-261, Birkhäuser, Basel (2004).

[12] J.-P. Serre, Modular forms of weight one and Galois representations, in Algebraic number fields: $L$-functions and Galois properties (Proc. Sympos., Univ. Durham, Durham, 1975), 193-268, Academic Press, London (1977).

[13] _ Local fields, Vol. 67 of Graduate Texts in Mathematics, Springer-Verlag, New York (1979). 
[14] - L'invariant de Witt de la forme $\operatorname{Tr}\left(x^{2}\right)$, Comment. Math. Helv. 59 (1984), no. $4,651-676$.

Centre for Mathematics and its Applications, Mathematical Sciences Institute Australian National University, Canberra, ACT 0200, Australia.

E-mail address: debargha.banerjee@anu.edu.au

School of Mathematics, TIFR, Homi Bhabha Road, Mumbai 400005, India.

E-mail address: eghate@math.tifr.res.in 